\title{
OPEN The behavior of residual tumors following incomplete surgical resection for vestibular schwannomas
}

\author{
Hun Ho Park ${ }^{1}$, So Hee Park $2,3,4,5$, Hyeong-Cheol Oh ${ }^{1}$, Hyun-Ho Jung 2,3,4,5, \\ Jong Hee Chang ${ }^{2,3,4,5}$, Kyu-Sung Lee ${ }^{1}$, Won Seok Chang ${ }^{2,3,4,5}$ \& Chang-Ki Hong ${ }^{1 \bowtie}$
}

The management of vestibular schwannoma (VS) with residual tumor following incomplete resection remains controversial and little is known regarding postoperative tumor volume changes. The behavior of residual tumors was analyzed for 111 patients who underwent surgery for newly diagnosed VS between September 2006 and July 2017. The postoperative tumor volume changes were assessed during a mean follow-up of 69 months (range 36-147 months). Fifty-three patients underwent imaging surveillance following incomplete resection. There was no residual tumor growth in 44 patients (83\%). A significant regression of residual tumor volume was noted in the no growth group at postoperative 1 year $(p=0.028), 2$ years $(p=0.012)$, but not from 3 years onwards. Significant predictors of regrowth were immediate postoperative tumor volume $\geq 0.7 \mathrm{~cm}^{3}$ (HR 10.5, $\left.p=0.020\right)$ and residual tumor location other than the internal auditory canal (IAC) (HR 6.2, $p=0.026)$. The mean time to regrowth was 33 months (range 5-127 months). The 2-, 5-, and 10-year regrowth-free survival rates were $90.6 \%, 86.8 \%$, and $83 \%$, respectively. In conclusion, significant residual tumor regression could occur within 2 years for a VS with an immediate postoperative tumor volume less than $0.7 \mathrm{~cm}^{3}$ or residual tumor in IAC.

The goal of vestibular schwannoma (VS) treatment is to achieve long-term tumor control and preserve neurological function. Treatment modalities include conservative management, microsurgery, radiosurgery or combination of both micro- and radiosurgery. Surgical resection remains the primary treatment that can provide the greatest chance of a long-term cure, especially for large, symptomatic tumors ${ }^{1}$. However, the narrow surgical corridor of the cerebellopontine angle and the intimate relationship of VS with delicate neurovascular structures such as the facial nerve have resulted in $30-50 \%$ rates of facial nerve dysfunction with complete resection ${ }^{2-5}$. Recent studies have shown that a planned incomplete resection followed by upfront gamma knife radiosurgery (GKRS) could achieve both satisfactory tumor control and neurological outcome ${ }^{6-9}$. Unfortunately, incomplete resection carries the risks of tumor regrowth and higher radiation-dose to reduce the risks of regrowth that could lead to tumor swelling, cerebral edema, and cranial nerve neuropathy ${ }^{9-13}$. The inherent, additional surgical risks must also be taken into account should GKRS fail to control the tumor ${ }^{14}$. The question of when and under what circumstances should GKRS be performed following incomplete resection will depend on the incidence and the rate of tumor regrowth, and the benefit on neurological outcome. Several studies in the past have focused on the long-term natural history of VS, but little is known about the long-term behavior of residual VS following incomplete surgical resection. In this study, we report a long-term analysis of patients who underwent surgery for newly diagnosed VS. We analyze the behavior of residual tumors after incomplete resection by measuring the sequential postoperative tumor volume changes. We also assess the neurological outcome, especially the facial nerve function. The purpose of the study is not to support one management approach over another, but to better understand the behavior of residual VS following incomplete resection.

\footnotetext{
${ }^{1}$ Department of Neurosurgery, Gangnam Severance Hospital, Yonsei University Health System, Seoul, Republic of Korea. ${ }^{2}$ Department of Neurosurgery, Severance Hospital, Yonsei University Health System, Seoul, Republic of Korea. ${ }^{3}$ Gamma Knife CenterSeverance Hospital, Yonsei University Health System, Seoul, Republic of Korea. ${ }^{4}$ Brain Tumor Center, Severance Hospital, Yonsei University Health System, Seoul, Republic of Korea. ${ }^{5}$ Brain Research Institute, Severance Hospital, Yonsei University Health System, Seoul, Republic of Korea. ${ }^{\varpi}$ email: yedamin@ yuhs.ac
} 


\section{Methods}

A long-term, retrospective, and consecutive analysis was performed on 111 patients who underwent surgical resection for newly diagnosed VS from September 2006 to July 2017. The data of 53 patients who had residual tumor following incomplete surgical resection were put on imaging surveillance for the residual tumor. Patients with radiologic and clinical follow-up of 3 years or longer matched the inclusion criteria of this study. There were no lost to follow-up among patients who had residual tumor following incomplete resection. The decision to observe rather than to undergo an upfront GKRS was made depending on the residual tumor volume, functional outcome, and patient's preference after informed consent. The data was gathered and analyzed after obtaining informed consent from every patient. The study was approved by the Institutional Review Board of Gangnam Severance Hospital Human Research Protection Center and conducted in accordance with the ethical guidelines of the Declaration of Helsinki.

Data interpretation. All patients underwent magnetic resonance imaging (MRI) including T2-weighted and gadolinium enhanced T1-weighted axial, coronal, and sagittal images, acquired at $1 \mathrm{~mm}$ slice thickness. A scheduled imaging surveillance protocol with gadolinium enhanced MRI was performed for 15 years from the day of the surgery. The imaging studies were obtained before surgery, 3 months after surgery (immediate), annually for the first 3 years, and every 2 to 3 years thereafter. Pre- and postoperative tumor volumes were assessed by two neurosurgeons using Leksell GammaPlan software version 8.0 (Elekta AB). All performed images were also interpreted by two neuroradiologists who were blinded to patient information. Gross-total resection (GTR) was defined as complete tumor removal confirmed by the surgeon intraoperatively with no evidence of residual tumor on immediate and 1 year postoperative MRIs. Near-total resection (NTR) was defined as above 95\% tumor resection with a thin layer of residual tumor on a neurovascular structure, confirmed by the surgeon intraoperatively, and on immediate and 1 year postoperative MRIs. Subtotal resection (STR) and partial resection (PR) were defined as $90-95 \%$ and less than $90 \%$ tumor resection, confirmed by the surgeon intraoperatively, and on immediate and 1 year postoperative MRIs, respectively. Tumor regrowth was defined as residual, enhancing, solid tumor volume increase of more than $20 \%$ compared to the first postoperative MRI with persistent growth on consecutive scans ${ }^{15}$. Neurological outcome was assessed using House-Brackmann (HB) classification for facial and Gardner-Robertson Hearing Scale (GR) for cochlear nerve function.

Surgical procedure. The choice of surgical approach depended on the size, location, surgical line-of-sight, extent of the tumor, and surgeon's preference. The facial nerve was routinely monitored and localized using facial nerve electromyography and stimulator throughout the surgical procedure. The cochlear nerve was monitored using intraoperative brainstem auditory evoke potentials if the patient had serviceable hearing. The cisterna magna was opened to relieve intracranial pressure and minimize cerebellar retraction. The roof of the internal auditory canal (IAC) was drilled to remove the tumor and decompress the canal to avoid retraction injury to the nerves before intracapsular debulking was performed with an ultrasonic aspirator. The facial nerve was first identified at the meatal segment, and then at the root entry zone to perform both antero- and retrograde dissection of the nerve. The intention of the surgery was a GTR, but an incomplete resection was performed if the tumor capsule was adherent to the facial nerve.

Statistical analysis. Tumor characteristics, size, and volume changes were analyzed and compared between the no growth and regrowth group using Mann-Whitney $U$ test. A Fischer's exact test was used to compare categorical variables. Univariate logistic regression analysis was conducted to determine significant predictors of regrowth. A Cox proportional hazards model was used to report hazard ratios of significant factors with 95\% confidence intervals. A stepwise multivariate logistic regression analysis was also performed to seek any independent predictors of regrowth. Regrowth-free survival was calculated using the Kaplan-Meier method. Data analyses were performed using SPSS software (version 18.0, SAS Institute Inc., Chicago, IL, USA). Graphs depicting long-term tumor volume changes and facial nerve outcome at last follow-up were plotted using Microsoft Excel software (version 16.0, Microsoft Inc., Redmond, WA, USA). Two-tailed and p values of $\leq 0.05$ were considered statistically significant.

\section{Results}

Patient and tumor characteristics. One hundred and eleven patients underwent surgery for newly diagnosed VS, in which 51 (45.9\%) achieved GTR with no recurrence during the study period (Fig. 1). Among 60 patients (54.1\%) with residual tumor following surgical resection, 7 patients underwent upfront GKRS and 53 patients were put on imaging surveillance for the residual tumors. Of the 53 patients with incomplete resections, there were 36 female $(67.9 \%)$ and 17 male $(32.1 \%)$ patients with a mean age of 50 years (range 20-75 years) (Table 1). The mean tumor length, width, height, and volume were $3.4 \mathrm{~cm}$ (range 1.2-6.1 cm), $2.6 \mathrm{~cm}$ (range $0.8-4.5 \mathrm{~cm}), 2.6 \mathrm{~cm}$ (range $1.0-4.8 \mathrm{~cm}$ ), and $13.5 \mathrm{~cm}^{3}$ (range $0.7-42.7 \mathrm{~cm}^{3}$ ), respectively. All patients had tumors that were Koos grade III $(28.3 \%)$ or IV $(71.7 \%)$ with a solid $(64.2 \%)$ or cystic $(35.8 \%)$ character. The foremost symptom was hearing difficulty (75.5\%) with a GR class of I to V in $24.5 \%, 20.8 \%, 28.3 \%, 5.7 \%$, and $20.8 \%$ of patients, respectively. The rate of preoperative unserviceable hearing (GR classes III to V) was $54.8 \%$. The preoperative facial nerve function was mostly normal (94.3\%) with a HB grade I. There was 1 patient (1.9\%) with $\mathrm{HB}$ grade II and $2(3.8 \%)$ with HB grade IV facial nerve function. The mean radiologic and clinical follow-up was 69 months (range 36-147 months). The rate of 3-, 5-, 7-, and 10-year radiologic and clinical follow-up were $100 \%, 52.8 \%, 26.4 \%$, and $9.4 \%$, respectively. 


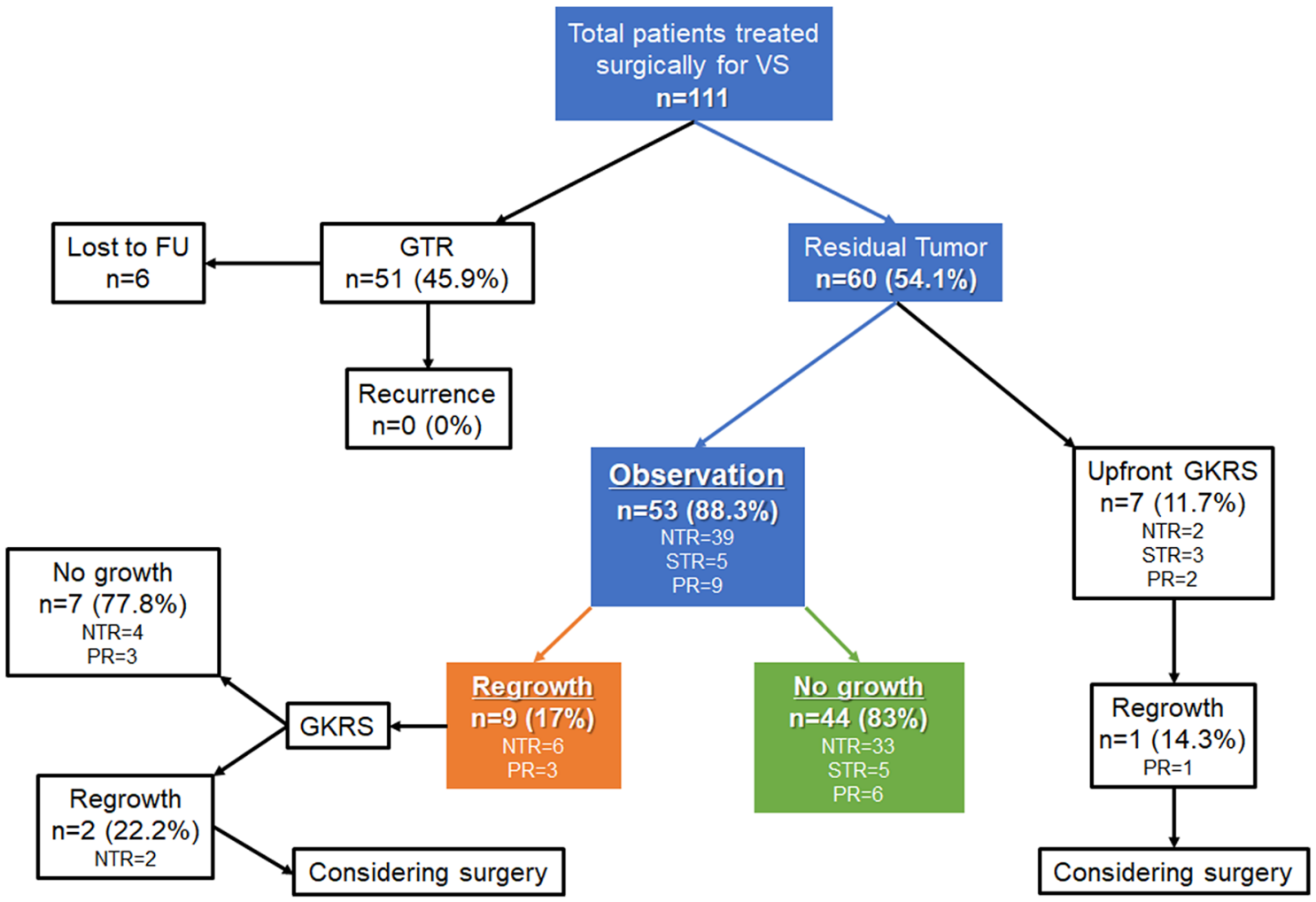

Figure 1. Flow chart showing the management and outcome of patients with residual tumor following incomplete resection.

Tumor control and radiologic outcome. Retrosigmoid lateral suboccipital (92.5\%) and presigmoid retrolabyrinthine posterior transpetrosal (7.5\%) approaches were performed for 53 patients with incomplete resections. NTR was achieved in 39 patients (73.6\%), STR in 5 patients (9.4\%), and PR in 9 patients (17\%). The mean extent of resection (EOR) for incomplete resections was $95.8 \%$ (range $77.8-99.8 \%$ ) with a mean immediate postoperative tumor volume of $0.36 \mathrm{~cm}^{3}$ (range $0.01-3.64 \mathrm{~cm}^{3}$ ). The most common site of residual tumor was IAC (83\%), followed by the brain stem (5.7\%), trigeminal nerve (5.7\%), brain stem and facial nerve (3.8\%), and facial nerve (1.9\%) (Fig. 2). There was no residual tumor growth in 44 patients (83\%) and regrowth in 9 patients (17\%) during the study period. There was significant reduction of tumor volume compared to the prior MRI scan in the no growth group at postoperative 1 year $(p=0.028)$ and 2 years $(p=0.012)$, but not from 3 years $(p=0.279)$ onwards (Fig. 3). The mean time from surgery to residual tumor growth in the regrowth group was 33 months (range 5-127 months), in which 8 patients underwent salvage GKRS and 1 patient underwent surgical resection (Fig. 4). The were 2 patients (22.2\%) with persistent residual tumor growth after GKRS. The mean regrowth-free period for VS with incomplete resections was 131 months (95\% CI 114-148 months). The regrowth-free survival at 2,5 , and 10 years was $90.6 \%, 86.8 \%$, and $83 \%$, respectively (Fig. $5 \mathrm{~A}$ ).

Predictors of tumor regrowth. There were no differences in patient and tumor characteristics such as age, sex, preoperative tumor size and volume, and Koos grade between the no growth and regrowth group (Table 2). The mean EOR of no growth and regrowth group was $96 \%$ and $95.2 \%$, respectively $(\mathrm{p}=0.903)$. The mean preoperative, immediate postoperative, 1-, 2-, 3-, 5-, 7-, 10-, and 13-year tumor volumes were $12.2 \mathrm{~cm}^{3}$, $0.27 \mathrm{~cm}^{3}, 0.19 \mathrm{~cm}^{3}, 0.14 \mathrm{~cm}^{3}, 0.14 \mathrm{~cm}^{3}, 0.12 \mathrm{~cm}^{3}, 0.08 \mathrm{~cm}^{3}$, and $0.11 \mathrm{~cm}^{3}$ in the no growth and $18.5 \mathrm{~cm}^{3}, 0.8$ $\mathrm{cm}^{3}, 0.69 \mathrm{~cm}^{3}, 1.04 \mathrm{~cm}^{3}, 1.21 \mathrm{~cm}^{3}, 1.41 \mathrm{~cm}^{3}, 1.95 \mathrm{~cm}^{3}$, and $0.35 \mathrm{~cm}^{3}$ in the regrowth group, respectively. On univariate analysis, significant predictors of regrowth were immediate postoperative tumor volume $\geq 0.7 \mathrm{~cm}^{3}(\mathrm{HR}$ 10.5, 95\% CI 1.445-76.289, $\mathrm{p}=0.020$ ) (Fig. 5B) and residual tumor location other than IAC (HR 6.2, 95\% CI $1.246-31.250, \mathrm{p}=0.026$ ) (Fig. $5 \mathrm{C}$ ), but no significance could be established on multivariate analysis.

Functional outcome and surgical complications. The postoperative facial nerve function at last follow-up was $\mathrm{HB}$ grades I to $\mathrm{V}$ in $50.9 \%, 26.4 \%, 17 \%, 3.8 \%$, and $1.9 \%$ of patients, respectively. Compared to the facial nerve status before surgery, $50.9 \%$ of patients had completely normal facial nerve function, $3.8 \%$ had no change, $28.3 \%$ had deterioration by 1 grade, $13.2 \%$ by 2 grades, $1.9 \%$ by 3 grades, and another $1.9 \%$ by 4 grades. The rates of optimal HB (grades 1 and 2) were $71.8 \%$ for NTR, $80 \%$ for STR, and $100 \%$ for PR (Fig. 6). Postoperatively, $7.5 \%$ of patients retained serviceable hearing, unserviceable hearing remained the same in $54.8 \%$, and $37.7 \%$ had deterioration. There were no differences in postoperative facial nerve function and hearing between the no growth and regrowth group. As for surgical morbidities, 5 of 44 patients (11.4\%) in the no growth group had neurological deterioration. Three patients with facial numbness had near-complete recovery at last follow- 


\begin{tabular}{|c|c|}
\hline Variable & Value \\
\hline Age in years (range) & $50(20-75)$ \\
\hline \multicolumn{2}{|l|}{ Sex (\%) } \\
\hline Male & $17(32.1)$ \\
\hline Female & $36(67.9)$ \\
\hline \multicolumn{2}{|l|}{ Tumor size in cm (range) } \\
\hline Length & $3.4(1.2-6.1)$ \\
\hline Width & $2.6(0.8-4.5)$ \\
\hline Height & $2.6(1.0-4.8)$ \\
\hline \multicolumn{2}{|l|}{ Koos grade (\%) } \\
\hline III & $15(28.3)$ \\
\hline IV & $38(71.7)$ \\
\hline \multicolumn{2}{|l|}{ Tumor character (\%) } \\
\hline Solid & $34(64.2)$ \\
\hline Cystic & $19(35.8)$ \\
\hline \multicolumn{2}{|l|}{ Preoperative HB grade (\%) } \\
\hline I & $50(94.3)$ \\
\hline II & $1(1.9)$ \\
\hline IV & $2(3.8)$ \\
\hline Preoperative tumor volume in $\mathrm{cm}^{3}$ (range) & $13.5(0.7-42.7)$ \\
\hline \multicolumn{2}{|l|}{ Extent of resection (\%) } \\
\hline NTR & 39 (73.6) \\
\hline STR & $5(9.4)$ \\
\hline PR & $9(17)$ \\
\hline Extent of resection in \% (range) & $95.8(77.8-99.8)$ \\
\hline \multicolumn{2}{|l|}{ Postoperative tumor volume in $\mathrm{cm}^{3}$} \\
\hline Immediate & $0.36 \pm 0.54$ \\
\hline 1 -year & $0.28 \pm 0.37$ \\
\hline 2-year & $0.31 \pm 0.51$ \\
\hline 3-year & $0.35 \pm 0.55$ \\
\hline 5-year & $0.54 \pm 1.11$ \\
\hline 7-year & $1.01 \pm 2.08$ \\
\hline 10-year & $0.20 \pm 0.22$ \\
\hline \multicolumn{2}{|l|}{ Site of residual tumor $(\%)$} \\
\hline IAC & $44(83)$ \\
\hline BS & $3(5.7)$ \\
\hline CN5 & $3(5.7)$ \\
\hline $\mathrm{FN}+\mathrm{BS}$ & $2(3.8)$ \\
\hline FN & $1(1.9)$ \\
\hline \multicolumn{2}{|l|}{ Postoperative HB grade (\%) } \\
\hline I & $27(50.9)$ \\
\hline II & $14(26.4)$ \\
\hline III & $9(17)$ \\
\hline IV & $2(3.8)$ \\
\hline $\mathrm{V}$ & $1(1.9)$ \\
\hline \multicolumn{2}{|l|}{ Surgical morbidity (\%) } \\
\hline None & $48(90.6)$ \\
\hline Facial numbness & $3(5.7)$ \\
\hline Diplopia & $2(3.8)$ \\
\hline Regrowth (\%) & $9(17)$ \\
\hline Surgery to regrowth time in months (range) & $33(5-127)$ \\
\hline Follow-up time in months (range) & $69(36-147)$ \\
\hline
\end{tabular}

Table 1. Summary of patients with incomplete surgical resection for vestibular schwannomas. BS brain stem, CN5 trigeminal nerve, FN facial nerve, $H B$ House-Brackmann, IAC internal auditory canal, NTR near-total resection, $P R$ partial resection, $S T R$ subtotal resection. ${ }^{\star}$ Values shown are mean. 

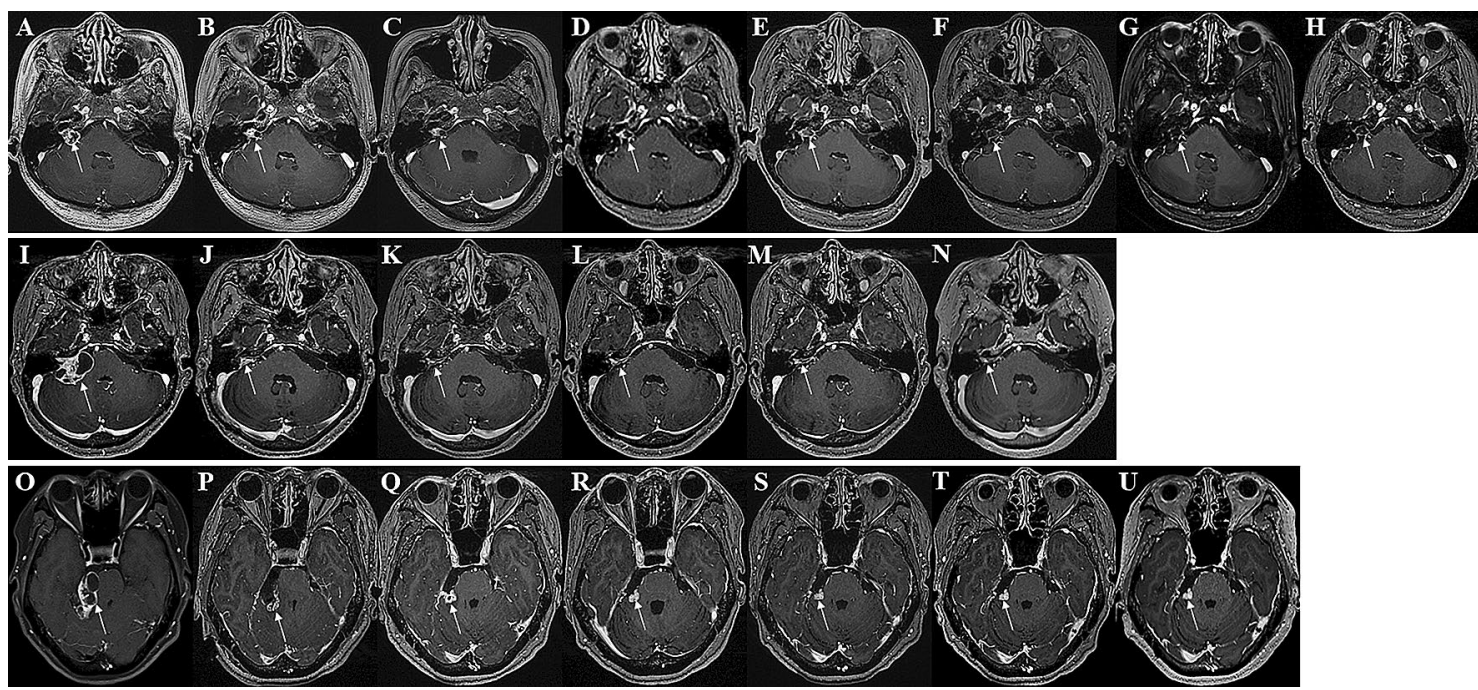

Figure 2. Preoperative (A,I,O), immediate (B,J,P), 1- (C,K,Q), 2- (D,L,R), 3- (E,M,S), 5- (F,N,T), 7- (G,U), and 10-year $(\mathbf{H})$ postoperative gadolinium enhanced T1-weighted axial MR images of patients with residual tumor (white arrows) following incomplete resection. (A-H) The residual tumor in IAC of a patient with partial resection (85.2\% extent of resection) gradually regressed from an immediate postoperative tumor volume of $1.19 \mathrm{~cm}^{3}(\mathbf{B})$ to $0.01 \mathrm{~cm}^{3}(\mathbf{H})$ over a follow-up of 147 months. The patient had a normal facial nerve function at last follow-up. (I-N) The residual tumor in IAC of a patient with subtotal resection ( $93 \%$ extent of resection) gradually regressed from an immediate postoperative tumor volume of $0.62 \mathrm{~cm}^{3}(\mathrm{~J})$ to $0.2 \mathrm{~cm}^{3}(\mathbf{N})$ over a follow-up of 62 months. The patient had a normal facial nerve function at last follow-up. (O-U) The residual tumor at the brain stem of a patient with near-total resection $(96.2 \%$ extent of resection) gradually regressed from an immediate postoperative tumor volume of $0.67 \mathrm{~cm}^{3}(\mathbf{P})$ to $0.38 \mathrm{~cm}^{3}$ (U) over a follow-up of 84 months. The patient had a facial nerve function of $\mathrm{HB}$ grade II at last follow-up.

up and the diplopia of 2 patients could be corrected with prism glasses. There were no surgical morbidities in the regrowth group.

\section{Discussion}

The management of large VSs has evolved over the last 30 years based on long-term tumor control and preservation of neurological function. Historically, a complete surgical resection carried the least risk of a long-term recurrence, but came at a 30-50\% risk of facial nerve dysfunction ${ }^{2-5,16}$. The concern that an aggressive surgical resection would cause inadvertent trauma to the facial nerve and other critical neurovascular structures has recently brought a shift in surgical practice. With advances in radiosurgical techniques, a planned incomplete resection followed by upfront GKRS has become a feasible treatment strategy that could achieve both satisfactory tumor control and neurological outcome ${ }^{6-9,17}$. However, an incomplete resection with upfront GKRS is not completely free from risks of its own such as pseudoprogression, cerebral edema, radiation injury to the brain stem, cranial nerve neuropathy, malignant transformation, and added surgical risks should GKRS fail ${ }^{9-14}$. Therefore, it is important to understand the behavior of residual tumors following an incomplete resection. Some authors showed that small, devascularized residual tumors do not grow ${ }^{18-20}$, while others proved that the proliferative activity of the tumor itself contributes to regrowth ${ }^{21-23}$. The question of whether the residual tumor will grow or not will depend on the pattern of regrowth, in which the incidence and rate of regrowth, and the benefit on neurological outcome need to be addressed. So far, the long-term behavior of residual tumors and the clinicopathologic factors that contribute to regrowth are not well understood. Therefore, we explored the long-term behavior of residual VS following incomplete resection, in terms of sequential tumor volume changes and assess the neurological outcome.

Definition of regrowth and extent of resection. The definition of two terminologies, regrowth and EOR has to be clarified before assessing the long-term behavior of residual tumors and the factors that affect them. There is currently no gold standard to define the growth of residual tumor in literature. Hence, many authors have traditionally determined regrowth as tumor size increase in its greatest dimension on follow-up MRIs. This could be imprecise if 2-dimensional diametric measurements are used since a true residual tumor size is difficult to compare from one examination to another, especially if the tumor is small. In this study, tumor regrowth was defined as residual, enhancing, solid tumor volume increase of more than $20 \%$ compared to the first postoperative MRI scan with persistent growth on consecutive scans ${ }^{7-9,15}$. Postoperative leptomeningeal and perineural remodeling can be difficult to distinguish from a true residual tumor or regrowth in early postoperative MRI scans $s^{15,24,25}$, therefore the first postoperative scan was performed at postoperative 3 months with 3-dimensional volumetric determination for every scan. The same principles were applied to determine EOR by measuring the volume of residual tumor on postoperative 3 month MRI ${ }^{24,25}$. The surgeon's subjective intraopera- 


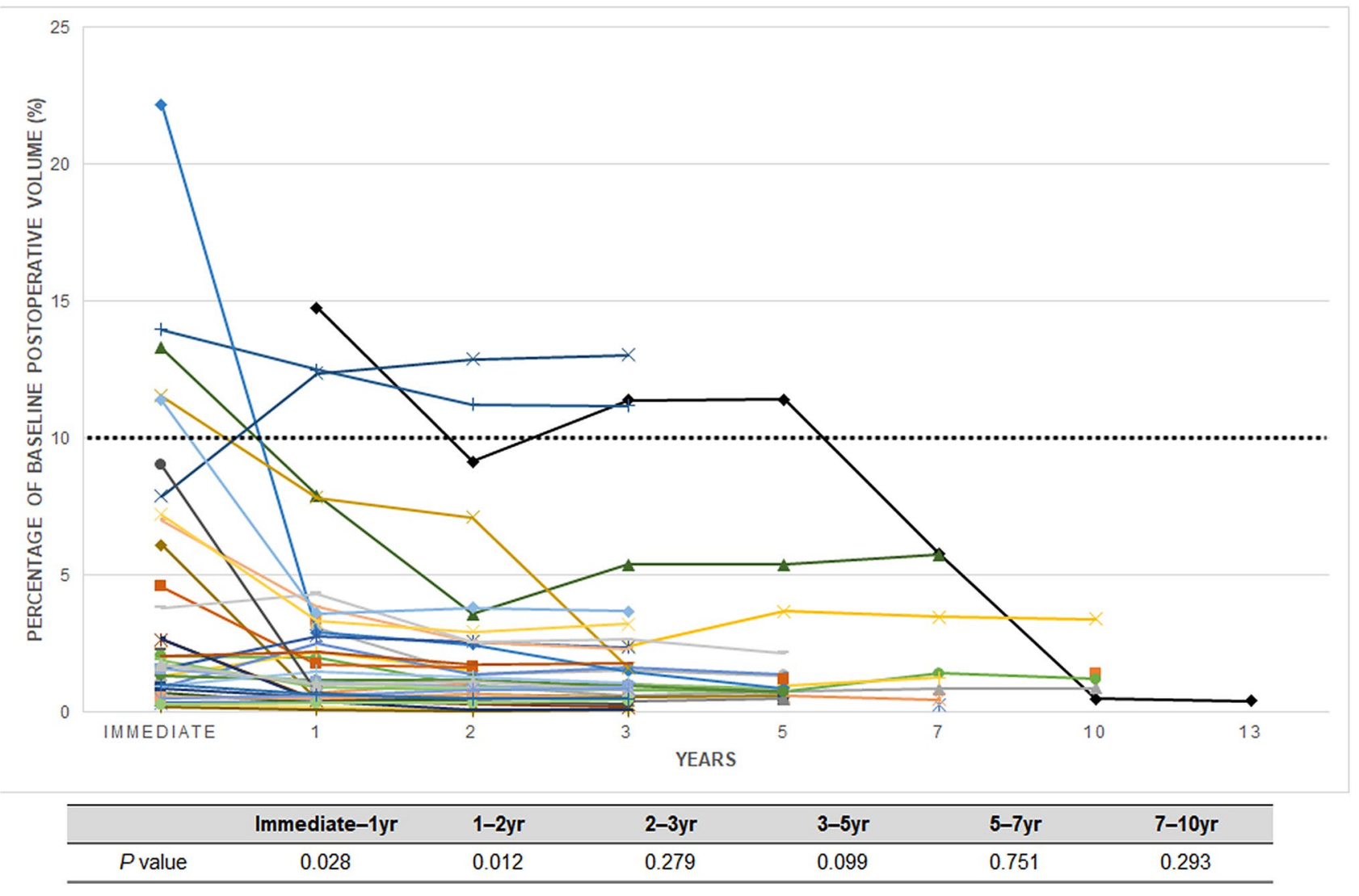

Figure 3. Graph outlining long-term tumor volume changes of patients with no residual tumor growth after incomplete resection. The y axis indicates the percentage of residual tumor volume compared to the preoperative tumor volume. There was significant reduction of tumor volume compared to the prior MRI scan at postoperative 1 year $(\mathrm{p}=0.028)$ and 2 years $(\mathrm{p}=0.012)$, but not from 3 years $(\mathrm{p}=0.279)$ onwards.

tive observation of EOR was also added to our definition considering the fact that not all residual tumors appear on early postoperative MRI scans, especially after NTR.

Behavior of residual tumors. The reported incidence of regrowth in large series that observed the behavior of residual VS after incomplete resection ranged from 6 to $21 \%$, which was consistent with $17 \%$ of this study (Table 3). The reported mean interval between surgery and regrowth was 33-47 months with a range between 7.2 and 108 months compared to 33 months (range 5-127 months) of the present series ${ }^{17,26-33}$. Considering the benign, slow-growing nature of VS, a long-term radiologic follow-up is necessary to validate the rate and interval of incidence. The present series underwent a scheduled imaging surveillance protocol for a mean 69 months (range 36-147 months). No patients were lost to follow-up with a 3-, 5-, 7-, and 10-year radiologic follow-up of $100 \%, 52.8 \%, 26.4 \%$, and $9.4 \%$, respectively. The patients with prior micro- or radiosurgery were excluded from the analysis to understand the true behavior of residual VS following incomplete resection. According to the long-term observation of the present and other studies, patients with incomplete resections are subjected to an imaging surveillance for at least 10 years with a regrowth peak around 3 years after surgery. During the observation period, residual tumors that did not grow had significant regression of tumor volume compared to the prior MRI scan at postoperative 1 year $(p=0.028)$ and 2 years $(p=0.012)$, but not from 3 years $(p=0.279)$ onwards, which was consistent with the findings of Akinduro et al. ${ }^{26}$. It can be postulated that surgical devascularization could lead to significant necrosis and shrinkage of the residual tumor for the first 2 years after surgery ${ }^{18-20}$. Hence, an imaging surveillance period of at least 2 years without further intervention could be suggested considering the inherent risks of upfront $\mathrm{GKRS}^{9-14}$.

Predictors of regrowth. Several factors including $\mathrm{EOR}^{16,17,28}$, residual tumor volume ${ }^{17,25,27,34-36}$, and the proliferative activity of the tumor itself ${ }^{21-23}$ have been reported to contribute to regrowth after incomplete resection of VS, but still, little is known about the significant clinicopathological factors ${ }^{24,26,29-33}$. In this study, an immediate postoperative tumor volume $\geq 0.7 \mathrm{~cm}^{3}$ had almost an 11 -fold greater risk and residual tumor location other than IAC had a sixfold greater risk of a regrowth than tumors that were less than $0.7 \mathrm{~cm}^{3}$ or in IAC. Therefore, residual tumors with a tumor volume less than $0.7 \mathrm{~cm}^{3}$ or in IAC could be possible candidates for imaging surveillance without further intervention. At least with the data in literature so far, residual tumor volumes exceeding $2 \mathrm{~cm}^{3}$ are not good candidates for observation ${ }^{17,25,34-36}$. There was no significance of EOR on regrowth 


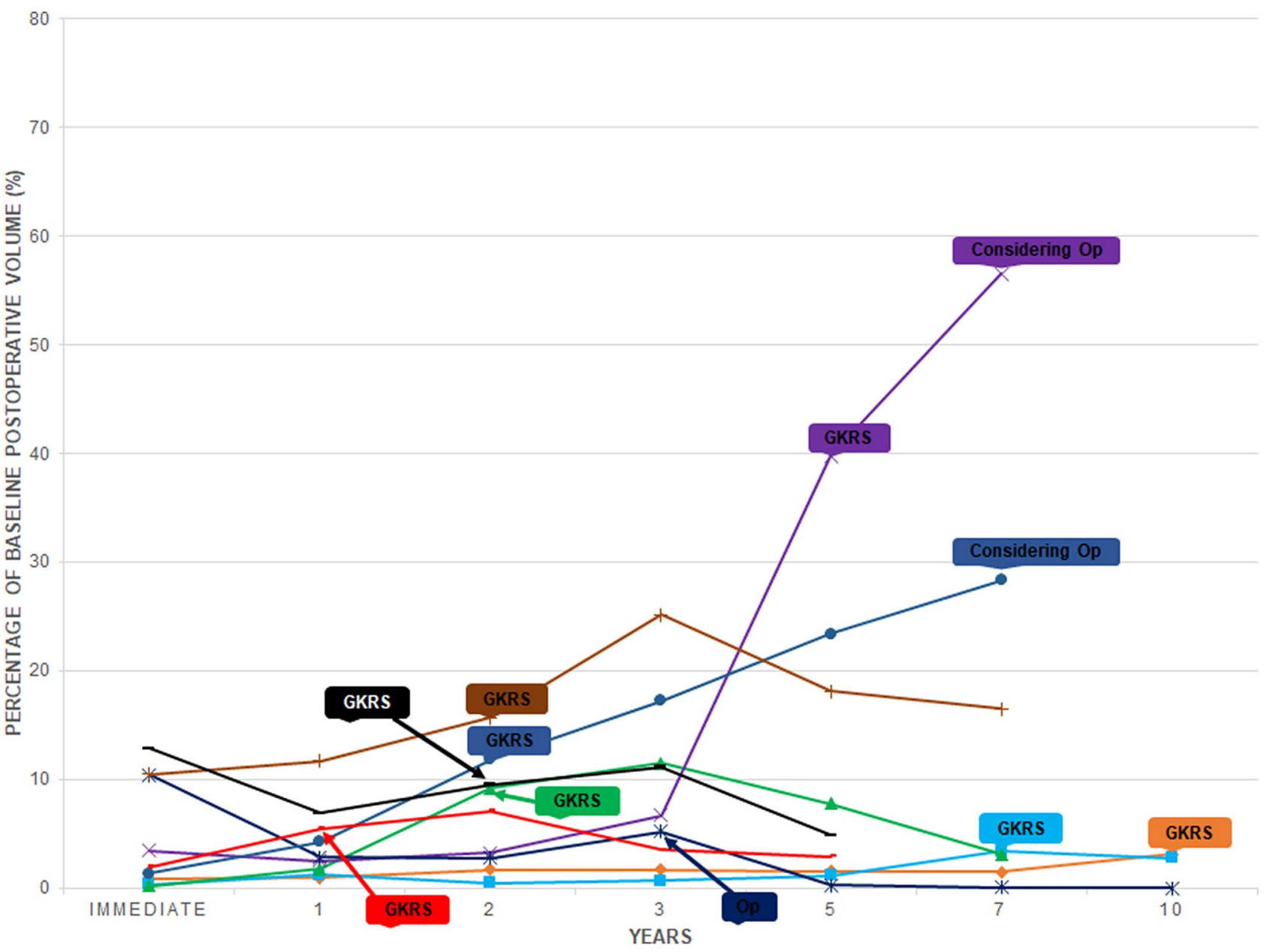

Figure 4. Graph outlining long-term tumor volume changes of patients with residual tumor growth after incomplete resection. The y axis indicates the percentage of residual tumor volume compared to the preoperative tumor volume. The mean time from surgery to residual tumor growth was 33 months (range 5-127 months). There were two patients with a late regrowth at postoperative 76 and 127 months. Among patients with residual tumor growth, 8 patients underwent salvage GKRS and 1 patient underwent salvage surgical resection. There were 2 patients (22.2\%) with persistent residual tumor growth after GKRS and are under consideration for a salvage surgical resection.
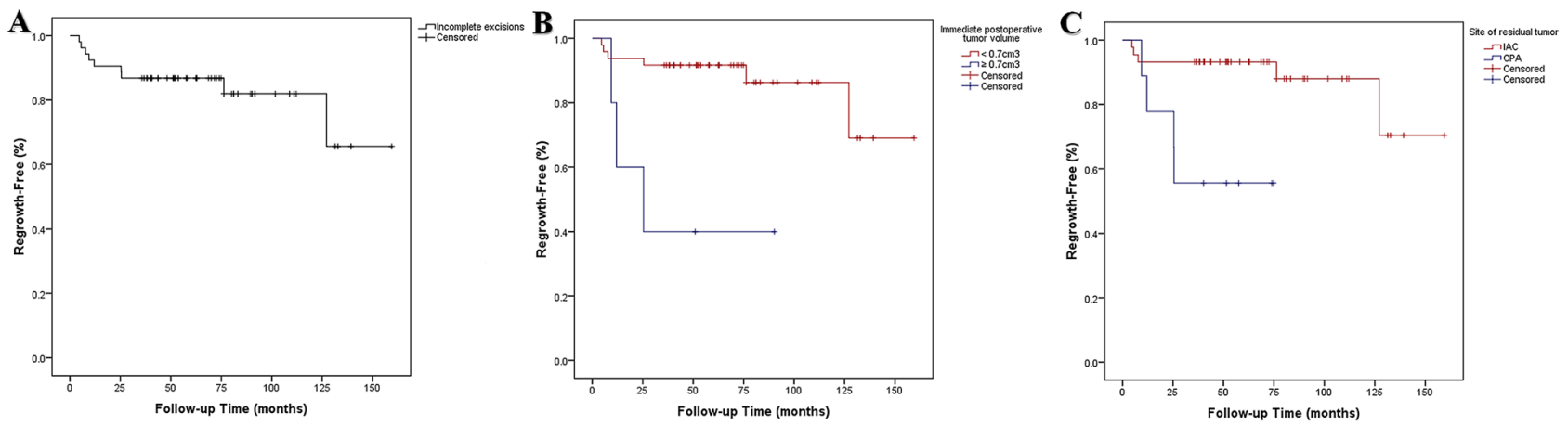

Figure 5. Kaplan-Meier curves of regrowth-free period following incomplete resection (A) and dichotomized for immediate postoperative tumor volume (B), and site of residual tumor (C).

in the present series. This could be due to the higher mean EOR for incomplete surgical resections (95.8\%) and lower mean immediate postoperative tumor volume $\left(0.36 \mathrm{~cm}^{3}\right)$ than other reported series ${ }^{17,26-31,33,34}$. The exclusion of patients with GTR in our analysis could have also contributed to the insignificance of EOR on regrowth. 


\begin{tabular}{|c|c|c|c|}
\hline & No growth & Regrowth & $P$ \\
\hline Patients & 44 & 9 & \\
\hline Age (years)* & $50 \pm 13.6$ & $49.7 \pm 11.2$ & 0.820 \\
\hline Sex, M/F & $13 / 31$ & $4 / 5$ & 0.445 \\
\hline \multicolumn{4}{|l|}{ Tumor size $(\mathrm{cm})^{*}$} \\
\hline Length & $3.3 \pm 1.2$ & $4 \pm 1$ & 0.064 \\
\hline Width & $2.6 \pm 0.9$ & $2.9 \pm 0.9$ & 0.312 \\
\hline Height & $2.5 \pm 0.9$ & $3.1 \pm 0.8$ & 0.064 \\
\hline \multicolumn{3}{|l|}{ Koos grade } & NA \\
\hline III & $15(34.1)$ & $0(0)$ & \\
\hline IV & $29(65.9)$ & $9(100)$ & \\
\hline \multicolumn{3}{|l|}{ Tumor character } & 0.706 \\
\hline Solid & $29(65.9)$ & $5(55.6)$ & \\
\hline Cystic & $15(43.1)$ & $4(44.4)$ & \\
\hline \multicolumn{3}{|l|}{ Preoperative HB grade } & NA \\
\hline I-II & $42(95.5)$ & $9(100)$ & \\
\hline III-V & $2(4.5)$ & $0(0)$ & \\
\hline Preoperative tumor volume $\left(\mathrm{cm}^{3}\right)$ & $12.2 \pm 9.9$ & $18.5 \pm 12.3$ & 0.128 \\
\hline \multicolumn{3}{|l|}{ Extent of resection } & 0.684 \\
\hline NTR & $33(75)$ & $6(66.7)$ & \\
\hline STR + PR & $11(25)$ & $3(33.3)$ & \\
\hline Extent of resection $(\%)^{*}$ & $96 \pm 4.9$ & $95.2 \pm 5.2$ & 0.903 \\
\hline \multicolumn{3}{|c|}{ Immediate postoperative tumor volume $\left(\mathrm{cm}^{3}\right)$} & 0.030 \\
\hline$<0.7$ & $42(95.5)$ & $6(66.7)$ & \\
\hline$\geq 0.7$ & $2(4.5)$ & $3(33.3)$ & \\
\hline \multicolumn{3}{|l|}{ Site of residual tumor } & 0.035 \\
\hline IAC & 39 (88.6) & $5(55.6)$ & \\
\hline $\mathrm{CPA}$ & $5(11.4)$ & $4(44.4)$ & \\
\hline \multicolumn{3}{|l|}{ Postoperative HB grade } & 0.665 \\
\hline I-II & $33(75)$ & $8(88.9)$ & \\
\hline III-V & $11(25)$ & $1(11.1)$ & \\
\hline
\end{tabular}

Table 2. Characteristic differences between no growth and regrowth group following incomplete surgical resection for vestibular schwannomas. $C P A$ cerebello-pontine angle, $H B$ House-Brackmann, $I A C$ internal auditory canal, NA not applicable, NTR near-total resection, $P R$ partial resection, STR subtotal resection. ${ }^{*}$ Values shown are mean $\pm \mathrm{SD}$.

Extent of resection and functional outcome. Among patients with incomplete resections for VS, optimal facial nerve outcomes (HB grades I and II) have been reported to be $51-84 \%$ for NTR and $55-100 \%$ for STR ${ }^{17,26-33}$. The postoperative facial nerve function of the present study was within the range of the previous reports, whereby $71.8 \%, 80 \%$, and $100 \%$ of NTR, STR, and PR had HB grades I and II, respectively. Although no statistical difference could be established in facial nerve outcome according to EOR the two factors seemed to have an inverse correlation. Nevertheless, the overall rate of no facial weakness at all (HB grade I) was lower than expected compared to other series ${ }^{17,26-33}$. This could be attributed to the larger preoperative tumor size and volume, and higher mean EOR than the reported series ${ }^{17,26-33}$. A larger tumor puts the facial nerve under tension which increases the likelihood of stretch injury and hypovascularization, resulting in poor facial nerve outcomes $s^{37,38}$. With regards to EOR, an inverse correlation between facial nerve outcome and EOR is not always true as long as care is taken to preserve the facial nerve ${ }^{28,29,31}$, which can also diminish as EOR increases. Surgical techniques such as IAC unroofing before tumor debulking, intracapsular tumor resection, and leaving behind any adherent tumor at the porus acousticus can minimize direct and indirect injury to the facial nerve. In the same sense, postoperative hearing will be retained as long as the anatomy and vascularity of the cochlear nerve is preserved $^{7-9}$.

Limitations. There are a small number of patients in each no growth and regrowth group, especially in the latter. Considering the benign, slow-growing nature of VS, regrowth is uncommon with 5- and 10-year regrowth-free survival rates reaching up to $85 \%$. Consequently, a long term follow-up is essential to validate the incidence, rate, and interval of regrowth. The present series underwent a long-term imaging surveillance with no patients lost to follow-up. However, a longer follow-up might be needed as two of our patients had a late regrowth at postoperative 76 and 127 months. The lack of standard norm for regrowth and EOR is another difficulty when it comes to validating the results and comparing them with other published reports. In this study, 


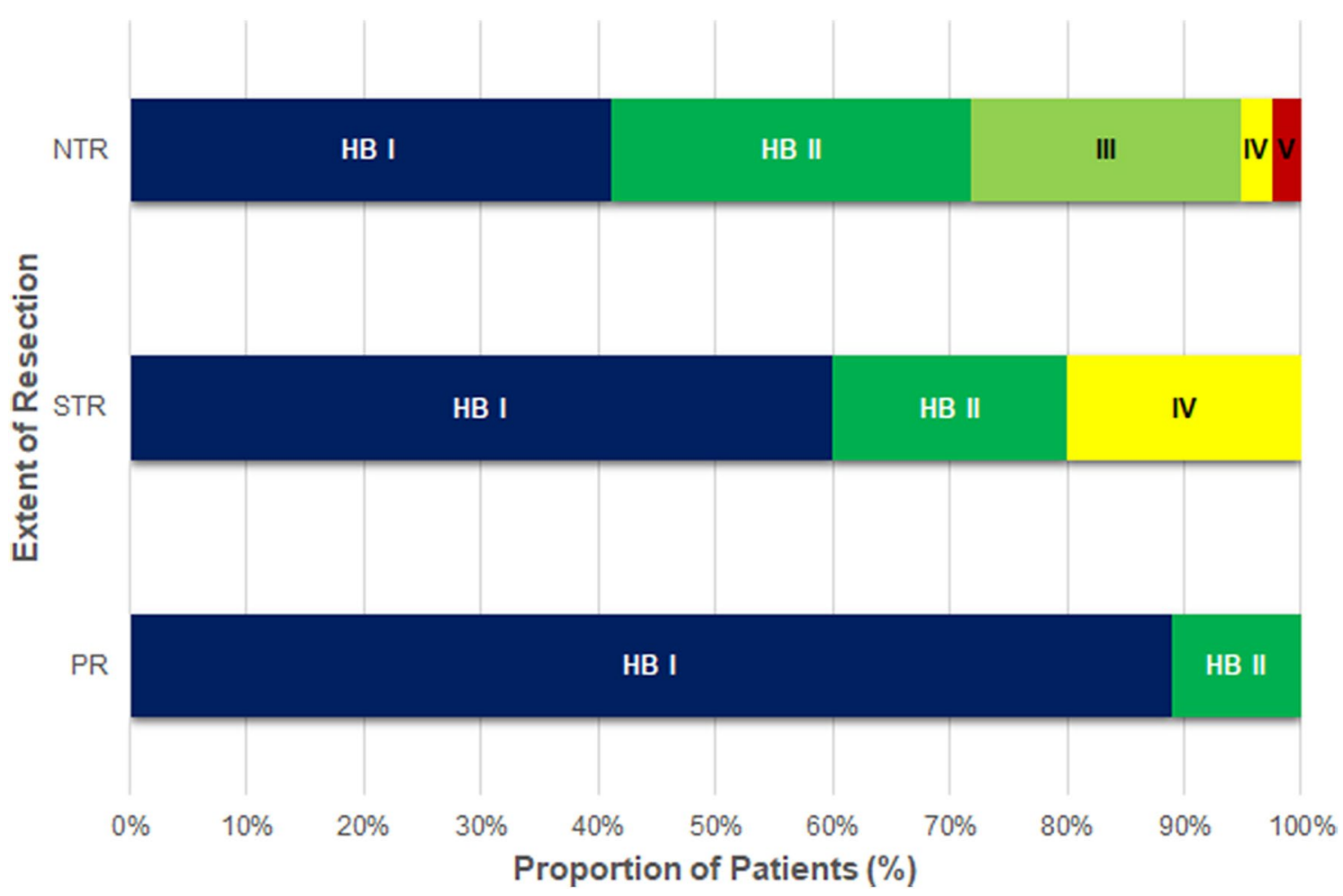

Figure 6. Graph summarizing facial nerve outcome at last follow-up according to extent of resection for vestibular schwannomas with incomplete resection. The rates of optimal $\mathrm{HB}$ (grades 1 and 2) were $71.8 \%$ for NTR, $80 \%$ for STR, and $100 \%$ for PR.

\begin{tabular}{|c|c|c|c|c|c|c|c|c|}
\hline Authors (year) & Pts (n) & Non-GTR (\%) & $\begin{array}{l}\text { Residual tumor } \\
\text { volume* }\left(\mathrm{cm}^{3}\right)\end{array}$ & $\begin{array}{l}\text { FN grade } 1 \text { and } \\
2(\%)\end{array}$ & Observation (\%) & $\begin{array}{l}\text { Regrowths among } \\
\text { observation (\%) }\end{array}$ & $\begin{array}{l}\text { Surgery to regrowth } \\
\text { time }^{\star}(\mathrm{mos})\end{array}$ & FU time ${ }^{\star}(\mathrm{mos})$ \\
\hline Haque et al. (2011) & 151 & $96 / 151(64)$ & NA & 97 & $96 / 96(100)$ & $20 / 96(21)$ & 47 & 72 \\
\hline Martin et al. (2012) & 212 & $65 / 212(31)$ & NA & 55 & $65 / 65(100)$ & $5 / 65(8)$ & NA & 66 \\
\hline Carlson et al. (2012) & 203 & $59 / 203(29)$ & NA & NA & $59 / 59(100)$ & $7 / 59(12)$ & NA & 42 \\
\hline Chen et al. (2014) & 111 & $111 / 111(100)$ & NA & 49 & $111 / 111(100)$ & $7 / 111(6)$ & 41 & 45 \\
\hline $\begin{array}{l}\text { Monfared et al. } \\
(2016)\end{array}$ & 73 & $61 / 73(84)$ & 1.1 & 81 & $61 / 61(100)$ & $13 / 61(21)$ & 35 & 38 \\
\hline Syed et al. (2016) & 450 & $42 / 450(9)$ & NA & 59 & $42 / 42(100)$ & $3 / 42(7)$ & NA & 73 \\
\hline Troude et al. (2018) & 159 & $143 / 159(90)$ & 0.56 & 84 & $66 / 143(46)$ & $12 / 66(18)$ & 37 & 59 \\
\hline Present study & 111 & $60 / 111(54)$ & 0.36 & 77 & $53 / 60(88)$ & $9 / 53(17)$ & 33 & 69 \\
\hline
\end{tabular}

Table 3. Literature review of large series investigating the behavior of residual tumors following incomplete surgical resection for vestibular schwannomas. FN facial nerve, FU follow-up, GTR gross-total resection, NA not applicable/available. ${ }^{\star}$ Values shown are mean and the percentages have been rounded up.

a scheduled imaging protocol, 3-dimensional volumetric determination, and an immediate postoperative MRI taken at 3 months were adopted to overcome the ambiguous definition of regrowth. The same principles were applied to the definition of EOR by adding the surgeon's subjective intraoperative observation of EOR as not all residual tumors appear on early postoperative MRI. The lack of data on the proliferative activity of VS is another weakness of this study since it has been reported to be a strong predictor of regrowth. The routine examination on the proliferative activity of the tumor had begun in the later stages of this study period and could not be included in the analysis. Finally, a comparison between observation and upfront GKRS for the residual tumors is beyond the scope of this study. The purpose of this study is not to support one management approach over another, but to share our findings of the behavior of residual VS after incomplete resection.

\section{Conclusion}

Significant residual tumor regression could occur within 2 years for a VS with an incomplete surgical resection. Residual tumors with a tumor volume less than $0.7 \mathrm{~cm}^{3}$ or in IAC could be possible candidates for imaging surveillance without further intervention. Regrowth peak around 3 years after surgery and patients with incomplete resections are subjected to an imaging surveillance for at least 10 years as late regrowth can occur. The conclusion 
that residual tumors could be observed rather than preceded with upfront GKRS should be carefully interpreted, taking into account the incidence of tumor regrowth and the benefit on neurological outcome.

Received: 8 October 2020; Accepted: 15 February 2021

Published online: 25 February 2021

\section{References}

1. Spielmann, P. M. \& Sillars, H. Assessing the threshold for vestibular schwannoma resection and the behavior of residual tumor. Otol. Neurotol. 34, 935-938 (2013).

2. Jung, S. et al. Current surgical results of retrosigmoid approach in extralarge vestibular schwannomas. Surg. Neurol. 53, 370-378 (2000).

3. Lanman, T. H., Brackmann, D. E., Hitselberger, W. E. \& Subin, B. Report of 190 consecutive cases of large acoustic tumors (vestibular schwannoma) removed via the translabyrinthine approach. J. Neurosurg. 90, 617-623 (1999).

4. Samii, M., Gerganov, V. M. \& Samii, A. Functional outcome after complete surgical removal of giant vestibular schwannomas: Clinical article. J. Neurosurg. 112, 860-867 (2010).

5. Zhang, X. et al. Facial nerve function after excision of large acoustic neuromas via the suboccipital retrosigmoid approach. J. Clin. Neurosci. 12, 405-408 (2005).

6. Fuentes, S. et al. Management of large vestibular schwannomas by combined surgical resection and gamma knife radiosurgery. Prog. Neurol. Surg. 21, 79-82 (2008).

7. Iwai, Y., Ishibashi, K., Watanabe, Y., Uemura, G. \& Yamanaka, K. Functional preservation after planned partial resection followed by gamma knife radiosurgery for large vestibular schwannomas. World Neurosurg. 84, 292-300 (2015).

8. Pan, H. C., Sheehan, J., Sheu, M. L., Chiu, W. T. \& Yang, D. Y. Intracapsular decompression or radical resection followed by gamma knife surgery for patients harboring a large vestibular schwannoma. J. Neurosurg. 117, 69-77 (2012).

9. Van de Langenberg, R. et al. Management of large vestibular schwannoma. Part I. Planned subtotal resection followed by gamma knife surgery: Radiological and clinical aspects. J. Neurosurg. 115, 875-884 (2011).

10. Chihara, Y., Ito, K., Sugasawa, K. \& Shin, M. Neurological complications after acoustic neurinoma radiosurgery: Revised risk factors based on long-term follow-up. Acta Otolaryngol. 127, 65-70 (2007).

11. Flickinger, J. C. et al. Acoustic neuroma radiosurgery with marginal tumor doses of 12 to 13 Gy. Int. J. Radiat. Oncol. Biol. Phys. 60, 225-230 (2004).

12. Ito, K., Shin, M., Matsuzaki, M., Sugasawa, K. \& Sasaki, T. Risk factors for neurological complications after acoustic neurinoma radiosurgery: Refinement from further experiences. Int. J. Radiat. Oncol. Biol. Phys. 48, 75-80 (2000).

13. Kim, J. H. et al. Predictive factors of unfavorable events after gamma knife radiosurgery for vestibular schwannoma. World Neurosurg. 107, 175-184 (2017).

14. Lee, H. J., Kim, M. J., Koh, S. H., Chang, W. S. \& Moon, I. S. Comparing outcomes following salvage microsurgery in vestibular schwannoma patients failing gamma-knife radiosurgery or microsurgery. Otol. Neurotol. 38(9), 1339-1344 (2017).

15. Van de Langenberg, R., de Bondt, B. J., Nelemans, P. J., Baumert, B. G. \& Stokroos, R. J. Follow-up assessment of vestibular schwannomas: Volume quantification versus two-dimensional measurements. Neuroradiology 51, 517-524 (2009).

16. Nakatomi, H. et al. Long-term risk of recurrence and regrowth after gross-total and subtotal resection of sporadic vestibular schwannoma. J. Neurosurg. 19, 1-7 (2017).

17. El-Kashlan, H. K., Zeitoun, H., Arts, H. A., Hoff, J. T. \& Telian, S. A. Recurrence of acoustic neuroma after incomplete resection. Am. J. Otol. 21, 389-392 (2000).

18. Kemink, J. L., Langman, A. W., Niparko, J. K. \& Graham, M. D. Operative management of acoustic neuromas: The priority of neurologic function over complete resection. Otolaryngol. Head Neck Surg. 104, 96-99 (1991).

19. Patni, A. H. \& Kartush, J. M. Staged resection of large acoustic neuromas. Otolaryngol. Head Neck Surg. 132, 11-19 (2005).

20. Silverstein, H., McDaniel, A., Norrell, H. \& Wazen, J. Conservative management of acoustic neuroma in the elderly patient. Laryngoscope 95, 766-770 (1985).

21. Aguiar, P. H. et al. Proliferative activity of acoustic neurilemomas without neurofibromatosis determined by monoclonal antibody MIB 1. Acta Neurochir. (Wien) 134, 35-39 (1995).

22. Bedavanija, A., Brieger, J., Lehr, H. A., Maurer, J. \& Mann, W. J. Association of proliferative activity and size in acoustic neuroma: Implications for timing of surgery. J. Neurosurg. 98, 807-811 (2003).

23. Iannella, G. et al. Subtotal resection of vestibular schwannoma: Evaluation with Ki-67 measurement, magnetic resonance imaging, and long-term observation. J. Int. Med. Res. 45, 1061-1073 (2017).

24. Fukuda, M., Oishi, M., Hiraishi, T., Natsumeda, M. \& Fujii, Y. Clinicopathological factors related to regrowth of vestibular schwannoma after incomplete resection: Clinical article. J. Neurosurg. 114, 1224-1231 (2011).

25. Vakilian, S., Souhami, L., Melançon, D. \& Zeitouni, A. Volumetric measurement of vestibular schwannoma tumour growth following partial resection: Predictors for recurrence. J. Neurol. Surg. B Skull Base 73, 117-120 (2012).

26. Akinduro, O. O. et al. Outcomes of large vestibular schwannomas following subtotal resection: Early post-operative volume regression and facial nerve function. J. Neurooncol. 143(2), 281-288 (2019).

27. Carlson, M. L. et al. Magnetic resonance imaging surveillance following vestibular schwannoma resection. Laryngoscope 122, 378-388 (2012).

28. Chen, Z. et al. The behavior of residual tumors and facial nerve outcomes after incomplete excision of vestibular schwannomas. J. Neurosurg. 120, 1278-1287 (2014).

29. Haque, R. et al. Efficacy of facial-sparing approach in patients with vestibular schwannomas. J. Neurosurg. 115, 917-923 (2011).

30. Martin, T. P. et al. Facial nerve outcomes in functional vestibular schwannoma surgery: Less than total tumour excision significantly improves results. J. Laryngol. Otol. 126, 120-124 (2012).

31. Monfared, A. et al. Facial nerve outcome and tumor control rate as a function of degree of resection in treatment of large acoustic neuromas: Preliminary report of the acoustic neuroma subtotal resection study (ANSRS). Neurosurgery 79, 194-203 (2016).

32. Syed, M. I. et al. The behavior of residual tumor after the intentional incomplete excision of a vestibular schwannoma: Is it such a bad thing to leave some behind?. Clin. Otolaryngol. 42(1), 92-97 (2017).

33. Troude, L. et al. Adjunctive gamma knife surgery or wait and scan policy after optimal resection of large vestibular schwannomas: Clinical and radiologic outcomes. World Neurosurg. 118, e895-e905 (2018).

34. Bloch, D. C., Oghalai, J. S., Jackler, R. K., Osofsky, M. \& Pitts, L. H. The fate of the tumor remnant after less-than-complete acoustic neuroma resection. Otolaryngol. Head Neck Surg. 113, 104-112 (2004).

35. Kameyama, S. et al. The long-term growth rate of residual acoustic neurinomas. Acta Neurochir. (Wien) 129, 127-130 (1994).

36. Ohta, S., Yokoyama, T., Nishizawa, S. \& Uemura, K. Regrowth of the residual tumor after acoustic neurinoma surgery. Br. J. Neurosurg. 12, 419-422 (1998).

37. Bloch, O. et al. Factors associated with preservation of facial nerve function after surgical resection of vestibular schwannoma. J. Neurooncol. 102, 281-286 (2011). 
38. Falcioni, M., Fois, P., Taibah, A. \& Sanna, M. Facial nerve function after vestibular schwannoma surgery. J. Neurosurg. 115, 820-826 (2011).

\section{Acknowledgements}

This study was supported by the National Research Foundation of Korea (NRF) grant funded by the government of Korea (No. 2020R1A2C200848011) and the Faculty Research Grant of Yonsei University College of Medicine (6-2019-0105).

\section{Author contributions}

Conception and design, H.H.P.; data collection, H.H.P., S.H.P. and H.C.O.; drafting the article, H.H.P.; provided clinical data, H.H.J., J.H.C., K.S.L., W.S.C. and C.K.H.; writing the manuscript, H.H.P.; critical revision of the article, C.K.H. All authors reviewed the manuscript.

\section{Competing interests}

The authors declare no competing interests.

\section{Additional information}

Correspondence and requests for materials should be addressed to C.-K.H.

Reprints and permissions information is available at www.nature.com/reprints.

Publisher's note Springer Nature remains neutral with regard to jurisdictional claims in published maps and institutional affiliations.

(c) (i) Open Access This article is licensed under a Creative Commons Attribution 4.0 International License, which permits use, sharing, adaptation, distribution and reproduction in any medium or format, as long as you give appropriate credit to the original author(s) and the source, provide a link to the Creative Commons licence, and indicate if changes were made. The images or other third party material in this article are included in the article's Creative Commons licence, unless indicated otherwise in a credit line to the material. If material is not included in the article's Creative Commons licence and your intended use is not permitted by statutory regulation or exceeds the permitted use, you will need to obtain permission directly from the copyright holder. To view a copy of this licence, visit http://creativecommons.org/licenses/by/4.0/.

(C) The Author(s) 2021 